\title{
Perineal recurrence of prostate ductal adenocarcinoma after transperineal brachytherapy: a case report and literature review
}

\author{
Takashi Fukagai, MD, PhD!, Kidai Hirayama, MD!, Masashi Morita, MD, PhD!, Kota Nishimura, MD', Jin Yamatoya, MD! \\ Tetsuo Noguchi, MD!, Yu Ogawa, MD, PhD!, Atsushi lgarashi, MD, PhD', Madoka Morota, MD, PhD², Masako Kato, MD³. \\ Kazuhiko Oshinomi, MD, PhD4, Yoshio Ogawa, MD, PhD4, Toyonori Tsuzuki, MD, PhD5 \\ 'Department of Urology, Showa University Koto Toyosu Hospital, Tokyo, Japan, ${ }^{2}$ Department of Radiation Oncology, Showa University Koto \\ Toyosu Hospital, Tokyo, Japan, ${ }^{3}$ Division of Radiation Oncology, Department of Radiology. Showa University School of Medicine, Tokyo, \\ Japan, ${ }^{4}$ Department of Urology, Showa University School of Medicine, Tokyo, Japan, ${ }^{5}$ Department of Surgical Pathology, Aichi Medical \\ University, Nagakute, Aichi, Japan
}

\begin{abstract}
Perineal recurrence after brachytherapy is an exceedingly rare complication. Moreover, ductal adenocarcinoma is a rare histological variant of prostate cancer. Herein, we describe a case of perineal recurrence from ductal adenocarcinoma of prostate after low-dose-rate brachytherapy (LDR-BT) in a 65-year-old male patient. The patient had localized prostate cancer, for which he received LDR-BT; however, he experienced perineal recurrence 2 years after receiving LDR-BT. Surgical excision was attempted, but we were unable to remove the whole tumor, owing to invasion to surrounding tissue. Pathological examination of resected tumor showed ductal adenocarcinoma of the prostate. External beam radiation therapy and high-dose-rate brachytherapy (HDR-BT) were performed for residual tumor. Mild mediastinal lymph node swelling was observed during clinical course of the disease. Hence, androgen deprivation therapy was administered with abiraterone after radiation therapy, and prostate-specific antigen level decreased to undetectable level. Biochemical failure after transperineal brachytherapy for prostate cancer should be considered as a perineal recurrence.

J Contemp Brachytherapy 2020; 12, 6: 612-617 DOI: https://doi.org/10.5114/jcb.2020.101696
\end{abstract}

Key words: prostate cancer, ductal adenocarcinoma, brachytherapy, perineal recurrence, neoplasm seeding.

\section{Purpose}

Low-dose-rate brachytherapy (LDR-BT) is a common standard therapy for localized prostate cancer [1]. Perineal recurrence after brachytherapy is an exceedingly rare complication. Perineal recurrence after perineal needle biopsies of the prostate occurs in up to $1 \%$ of patients with prostate cancer [2]. However, there are only a few reports on perineal recurrence after brachytherapy. Therefore, the characteristics and management of perineal recurrence after LDR-BT are not well-known.

Ductal adenocarcinoma (DAC) is a rare histological variant of prostate cancer, with a poorly understood natural history; the treatment for DAC is also not specified [3].

Therefore, herein, we report on the case of a patient with a perineal recurrence of prostate ductal adenocarcinoma after transperineal brachytherapy, and provide a literature review.

\section{Case report}

A primary care physician referred a 65-year-old man to another hospital owing to an elevated level of prostate-specific antigen (PSA; $7.38 \mathrm{ng} / \mathrm{ml}$ ) in October 2015. The patient underwent transrectal ultrasound-guided 12-core prostate biopsy in January 2016. He was diagnosed with prostatic adenocarcinoma of the right peripheral zones ( 2 cores), with a Gleason score of $3+4=7$. The tumor was diagnosed as stage cT2aN0M0 prostate cancer on the basis of findings from digital rectal examination, computed tomography (CT), magnetic resonance imaging (MRI), and bone scintigraphy. The patient elected to receive LDR-BT and was hence referred to our hospital, where he underwent LDR-BT in April 2016. The prostate volume was $31 \mathrm{ml}$. Iodine-125 loose seeds were used for LDR-BT with intra-operative dosimetry. A total of 70 seeds were transperineally inserted into the whole prostate using 16 needles under ultrasound guidance
Address for correspondence: Takashi Fukagai, MD, PhD, Department of Urology, Showa University Koto Toyosu Hospital, 5-1-38 Toyosu, Koto-ku, Tokyo 135-8577, Japan, phone: +81-362046514, fax: +81-362046998, « e-mail: fukagai@med.showa-u.ac.jp
Received: 11.07.2020

Accepted: 01.10 .2020

Published: 16.12.2020 
with a Mick applicator (Mick Radio-Nuclear Instruments, Inc., Bronx, NY, USA). The procedure was performed within 45 minutes, without any complications. Dosimetry with post-implant CT performed after 1 month showed that the prostate $\mathrm{D}_{90}$ was $186.49 \mathrm{~Gy}$, prostate $\mathrm{V}_{100}$ was $97.36 \%$, prostate $\mathrm{V}_{150}$ was $43.42 \%$, urethral $\mathrm{D}_{30}$ was $126.25 \%$, urethral $D_{5}$ was $139.15 \%$, rectal $V_{150}$ was $0.00 \mathrm{cc}$, and rectal $\mathrm{V}_{100}$ was $0.00 \mathrm{cc}$ of the prescribed dose of 160 Gy. After implanting the seeds, the PSA level decreased gradually, and the nadir was $0.4 \mathrm{ng} / \mathrm{ml}$ in April 2017. However, the PSA increased gradually, and it was 2.964 $\mathrm{ng} / \mathrm{ml}$ in January 2018. This value exceeded the Phoenix definition, indicating a possibility of biochemical failure [4]. Initially, we suspected that the increase in the PSA level was a bounce phenomenon and only observed the patient (i.e., no treatment was administered), but later on, the PSA level increased to $6.25 \mathrm{ng} / \mathrm{ml}$ and the patient complained of a right perineal mass with slight tenderness in April 2018. Pelvic MRI revealed a $2 \mathrm{~cm}$ perineal mass surrounded by the left obturator internus muscle, anal sphincter, and penile bulb (Figure 1). The radiologist diagnosed the mass as a small abscess, and we suspected an infection in the pelvis. Therefore, levofloxacin was administered to the patient for 2 weeks; however, the PSA level did not decrease, and the size of the mass increased to $3 \mathrm{~cm}$. Chest CT revealed mild mediastinal lymph node swelling, with a diameter of approximately $1 \mathrm{~cm}$, but these findings were not sufficient to make a diagnosis of metastasis of prostate cancer. We did not observe any other metastasis on CT and bone scans in August 2018. Accordingly, we discussed the treatment course with the patient, which included surgery, radiation, and androgen deprivation therapy (ADT). We attempted to excise the nodule as a part of the first step in September 2018. The margin of the nodule was not clear, and we suspected tumor invasion to the surrounding tissues, anal sphincter, and penile bulb. As we could not perform en bloc resection, we resected a part of the mass for histological diagnosis and placed titanium clips around the tumor location as a fiducial marker for radiation therapy. Histological examination of the lesion revealed an adenocarcinoma, similar to that previously identified on needle biopsy.
Immunostaining for PSA was positive in the resected tissue specimen. However, considering the unusual clinical course, we requested an expert urological pathologist (T.T.) to reevaluate the original prostate biopsy and the perineal recurrence. Both samples were diagnosed as DAC, a subtype of prostatic adenocarcinoma (Figure 2). After surgery, the PSA level decreased slightly but grew again just 1 month later. MRI performed in November 2018 revealed a tumor. We considered ADT as a concurrent treatment with radiation therapy. However, as the patient hoped to maintain his sexual function, we did not administer ADT. Moreover, we were concerned that the target for radiation therapy would be lost after tumor shrinkage due to ADT, despite placing the titanium clip markers. After reviewing the different radiation methods like stereotactic body radiotherapy (SBRT), we selected a high-dose-rate (HDR) boost to administer a high-dose of radiation directly to the tumor, and to avoid damaging the skin and other healthy tissue. The patient underwent a combination of external beam radiation therapy (EBRT) and HDR brachytherapy (HDR-BT) boost from December 2018 to February 2019. The perineal mass with the titanium clips as markers was identified using CT. This was the clinical target volume (CTV). The planning target volume (PTV) consisted of the perineal mass with the clips and 1-cm margins. The rectal wall, urethra, anal sphincter, and the previously treated prostate were considered as organs at risk (OARs). A total dose of $45 \mathrm{~Gy}$ in 25 fractions delivered with an energy of $10 \mathrm{MV}$, using 3 dimensional (3D) conformal radiotherapy/image-guided radiation therapy (Figure 3). An additional dose of $10 \mathrm{~Gy}$ in a single fraction was delivered to perineal mass with HDR-BT, 10 days after EBRT. The HDR-BT procedure was performed under spinal anesthesia. After the perineal mass under transrectal ultrasound (TRUS) in the lithotomy position was confirmed, flexible plastic needles with stylets were inserted into the perineal mass under TRUS guidance, freehand, without a template. After all the plastic needles were inserted, a CT using a 3D real-time planning system (Oncentra Brachy planning system, version 4.3, Elekta AB, Stockholm, Sweden) was performed. The perineal mass with titanium clips iden-
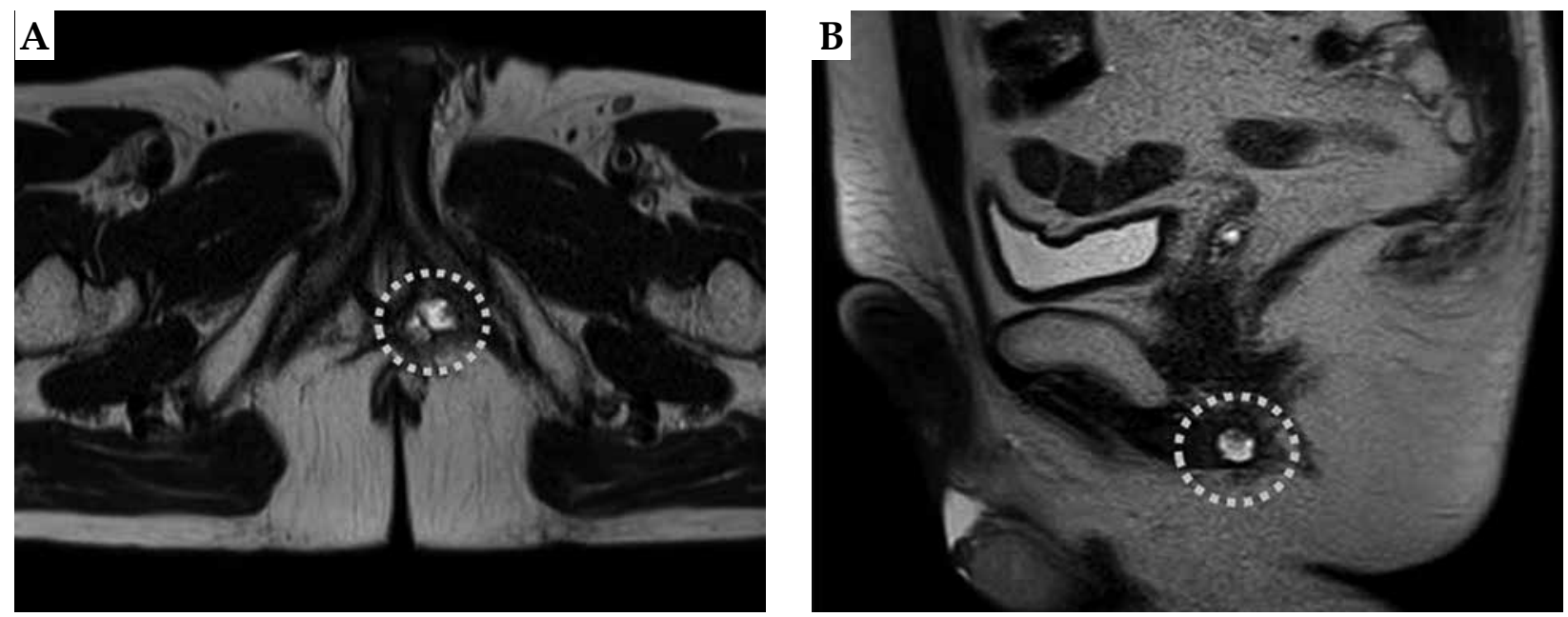

Fig. 1. Axial and sagittal T2-weighted pelvic magnetic resonance imaging scans showing a perineal lesion 

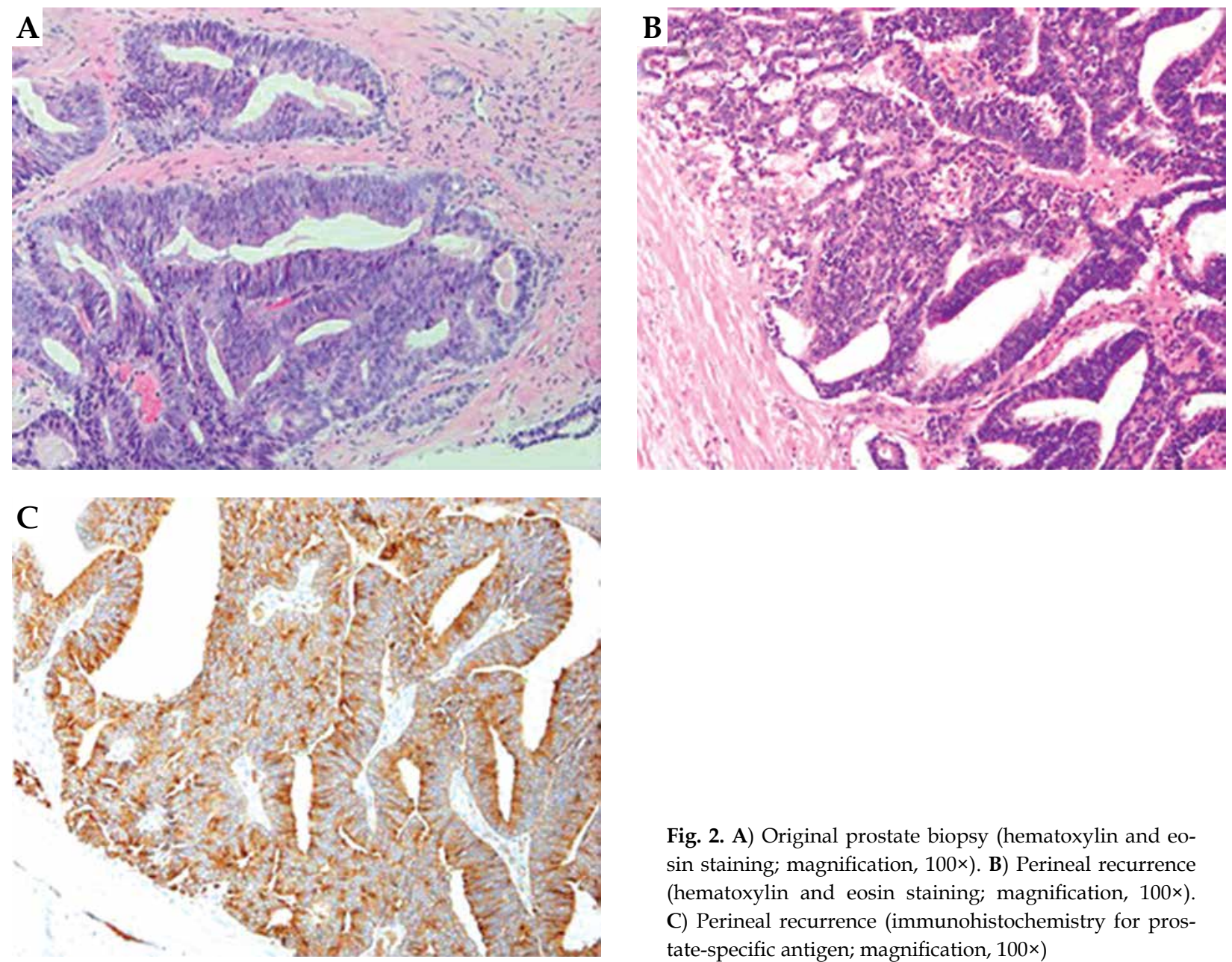

Fig. 2. A) Original prostate biopsy (hematoxylin and eosin staining; magnification, 100×). B) Perineal recurrence (hematoxylin and eosin staining; magnification, 100×). C) Perineal recurrence (immunohistochemistry for prostate-specific antigen; magnification, 100×)
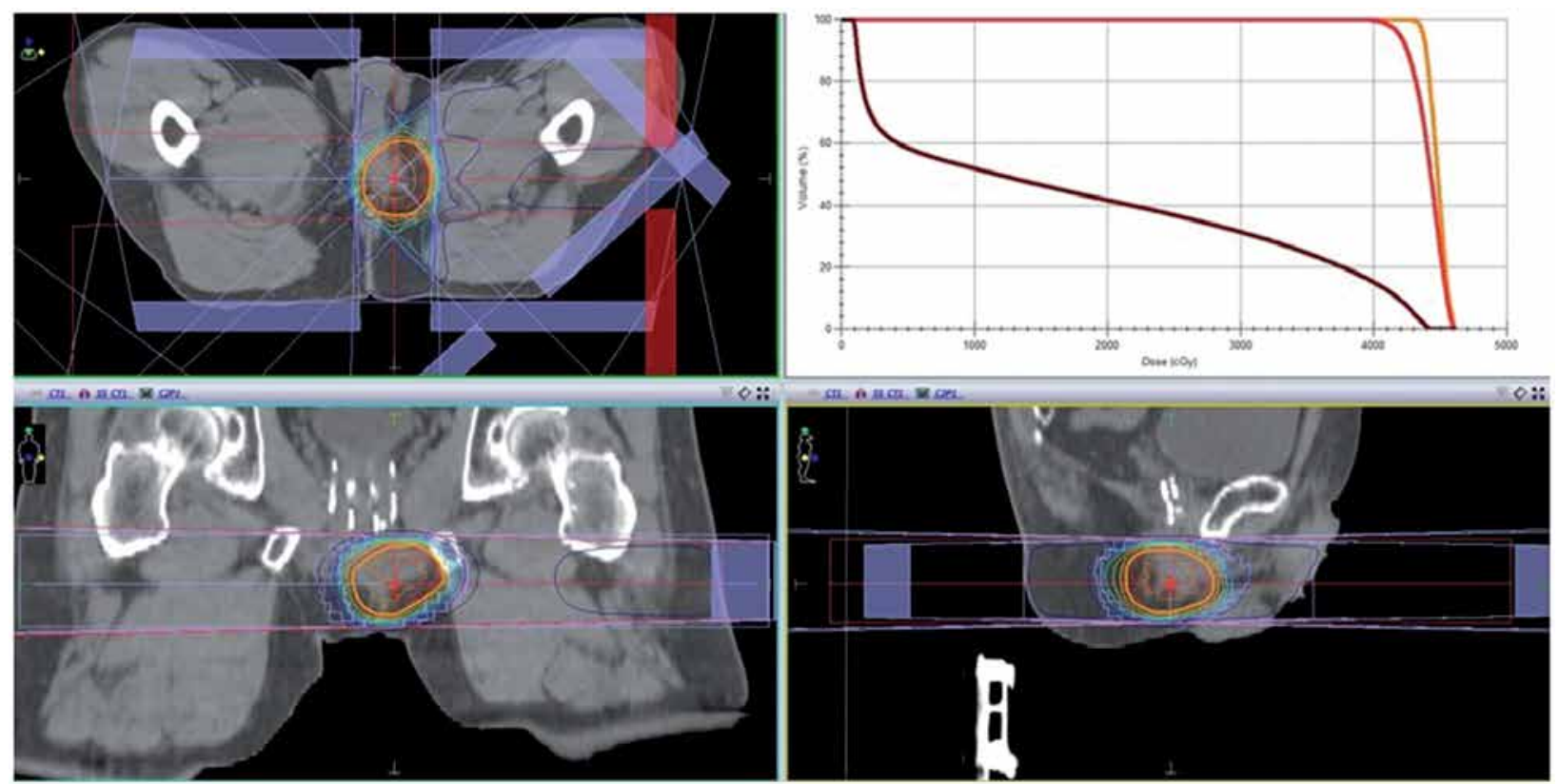

Fig. 3. Planning imaging and dose-volume histogram for the external beam radiation therapy plan

tified with CT was determined (CTV and EBRT). The whole perineal mass was contoured as the target volume, and the OARs included the rectum and urethra.
A geometrical optimization method was used, and the prescribed dose was 10 Gy to the entire perineal mass. Graphical optimization was used to optimize the distri- 


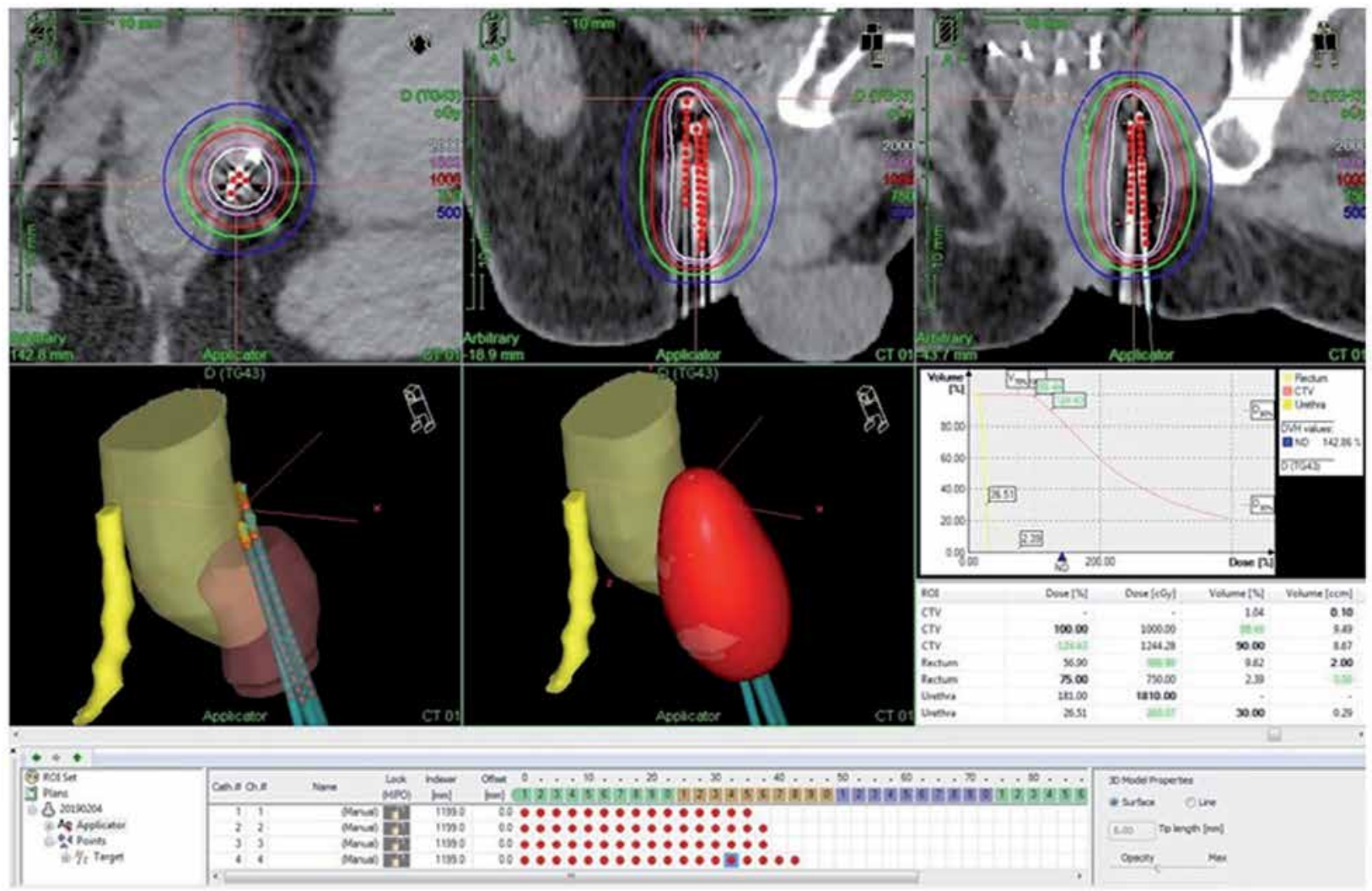

Fig. 4. Planning imaging and dose-volume histogram for the high-dose-rate brachytherapy plan

bution and to reduce the dose to the rectum. The treatment was delivered using a MicroSelectron HDR (Elekta $\mathrm{AB}$, Stockholm, Sweden) (Figure 4). With respect to skin toxicity from the treatment, the patient showed faint erythema and experienced burning sensation on the perineal skin. These symptoms were classified as grade 1 in the common toxicity criteria of adverse events (CTCAE) version 5. These symptoms were self-limiting without any treatment. While inserting the applicators for HDR-BT, we performed prostate biopsy to confirm local recurrence in the prostate after LDR-BT. However, no cancer cells were detected on the biopsy specimen from the prostate. Moreover, after the patient completed radiation therapy, the whole-body MRI conducted in March 2019 did not reveal any tumor in the perineal region. Nevertheless, the PSA level kept increasing and the mediastinal lymph node swelling increased to a diameter of $1.5 \mathrm{~cm}$. Finally, we started ADT with leuprolide acetate in March 2019. However, the PSA level still kept increasing. The treatment was changed to combination therapy with leuprolide acetate and abiraterone acetate in April 2019, after which the PSA level decreased rapidly and reached an undetectable range in December 2019. The present PSA value is $<0.008 \mathrm{ng} / \mathrm{ml}$, with no evidence of locoregional recurrence in 10 months (Figure 5). In addition, the mediastinal lymph nodes swelling almost disappeared.

\section{Discussion}

Perineal recurrence after brachytherapy is an exceedingly rare complication. The mechanism of perineal re-

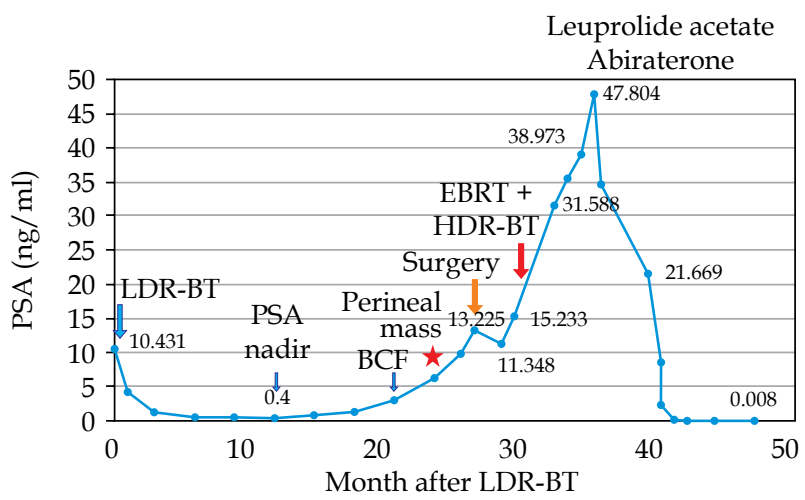

Fig. 5. Clinical course of the patient

$B C F$ - biochemical failure

currence is probable tumor cell seeding from the needle tract rather than local lymphogenous or hematogenous metastasis. The presented patient showed a nodule in the left perineal region, and a positive core on needle biopsy was from the left peripheral zone. In addition, the tumor location was almost consistent with the location of where the needle tract was injected for seed implantation, indicating that perineal recurrence developed due to tumor cell seeding from the needle tract. Moreover, tumor cell seeding along the needle tract is a rare complication after prostate biopsy. Volanis et al. reported that the incidence of tumor cell seeding after prostate biopsy was $<1 \%$ in 42 patients, with needle tract seeding mainly after transperineal biopsy [5]. However, that study included cases, 
in which biopsy was performed using 14-gauge Tru-Cut needles. In fact, the incidence of perineal recurrence is unclear after transrectal ultrasound-guided transperineal prostate biopsy performed via an 18-gauge needle. A literature search conducted using PubMed from inception until June 5, 2020, indicated 148 records. The literature search restricted the language to English and the search terms included "prostate cancer", "brachytherapy", and "perineal". Only three cases of perineal recurrence after brachytherapy were identified $[6,7,8]$. Hence, the incidence of perineal recurrence after brachytherapy is undetermined. A summary of all cases of perineal recurrence after brachytherapy including the present case is shown in Table 1 . The three previously reported patients did not experience any complications during the procedure, nor did they have any predisposing factors for needle tract seeding. In contrast, the presented patient had DAC of the prostate on pathological examination. Although poorly differentiated high-grade cancer is a risk factor of needle tract seeding $[9,10]$, the present literature review for needle tract seeding after prostate biopsy showed that the relation between needle tract seeding and tumor differentiation was not clear. In the present case, an expert urological pathologist diagnosed both the primary and recurrent tumor as DAC. While the characteristics of DAC are not well-known, DAC is rare and aggressive [11]. In addition, DAC metastasis has been observed in less common sites of metastasis of prostate cancer, such as the lungs and brain $[11,12,13]$ as well as the penis and testicles [14,15]. The present case showed mediastinal lymph node metastasis in addition to perineal recurrence. The perineal recurrence might have been induced owing to some unknown biological characteristics of DAC. Our case was the first case with DAC that showed a perineal recurrence after brachytherapy. The primary tumor in the prostate was under control with brachytherapy. Therefore, a DAC case is still a candidate for brachytherapy. But considering our unusual metastasis site, DAC patients who undergo brachytherapy should obtain a more careful follow-up for metastasis than usual cases. Moreover, the response of DAC to ADT is controversial; some studies showed that DAC responds well to ADT $[16,17]$, but the present case did not respond well to standard
ADT with leuprolide acetate. Consequently, we had to administer abiraterone acetate, a new strong androgen receptor inhibitor. Thus, the efficacy of ADT for DAC differs among cases. Most of the DAC cases were associated with conventional acinar adenocarcinoma [3]. Therefore, the efficacy of ADT for DAC might change depending on the proportion of acinar adenocarcinoma component.

The interval between brachytherapy and perineal recurrence varies greatly. The interval between brachytherapy and perineal recurrence in the three previously reported cases was 4 years, 6 years, and 11 years, respectively. In the present case, the interval was only 2 years between the initial treatment and the perineal recurrence. This early recurrence might be caused by the aggressive nature of DAC.

Various treatment strategies have been used for treating perineal recurrence after needle biopsy. The mostcommon approach was local excision, but excision of perineal recurrence was potentially toxic and highly invasive [5]. Owing to recent advances in radiotherapy techniques, small nodule-like perineal recurrences can be irradiated. A previouscase of perineal recurrence was treated withstereotactic volumetric modulated arc therapy (VMAT) [7]. In the present case, we attempted to excise the nodule via a transperineal approach to determine the histology, but the nodule was strongly adhered to the anal sphincter and penile bulb. Hence, we resected a part of the nodule for histological examination and placed titanium clips as a fiducial marker for radiation therapy. After surgery, we selected EBRT-boosted HDR-BT to administer high doses to the residual tumor. Then, ADT started after radiation therapy. In another previously reported case, the nodule had become too small after ADT to accurately place fiducial markers, and a CyberKnife ablation could not performed [6]. Therefore, the time of ADT initiation should be considered carefully in patients for whom radiation therapy is planned. Our strategy of treating the perineal metastasis of the patient with mediastinal lymph nodes metastasis before using ADT may be controversial. However, in this case, the patient noticed and experienced tenderness in the area of the perineal mass. Finally, the patient became free from these perineal symptoms after our treatment. It is probable that the applied ADT result-

Table 1. List of reports of perineal recurrence after brachytherapy for prostate cancer

\begin{tabular}{|c|c|c|c|c|c|c|c|c|c|c|c|c|}
\hline Reference & Year & Age & $\begin{array}{l}\text { iPSA } \\
(\mathrm{ng} / \mathrm{ml})\end{array}$ & cT & Histology & $\begin{array}{l}\text { Original } \\
\text { treat- } \\
\text { ments for } \\
\text { prostate }\end{array}$ & $\begin{array}{l}\text { Number } \\
\text { of seeds }\end{array}$ & $\begin{array}{c}\text { Number } \\
\text { of } \\
\text { needles }\end{array}$ & $\begin{array}{c}\text { Prescribe } \\
\text { dose }\end{array}$ & $\begin{array}{l}\text { PSA } \\
\text { nadir }\end{array}$ & $\begin{array}{l}\text { Time from } \\
\text { BT to } \\
\text { recurrence }\end{array}$ & $\begin{array}{l}\text { Treatments } \\
\text { for perineal } \\
\text { recurrence }\end{array}$ \\
\hline Teh et al. & 2001 & 68 & 36.7 & cT1c & $3+3=6$ & $\begin{array}{c}\text { BT (Gold) } \\
+ \text { EBRT }\end{array}$ & N.A. & N.A. & N.A. & 0.8 & 4 years & N.A. \\
\hline $\begin{array}{l}\text { Eppinag } \\
\text { et al. }\end{array}$ & 2014 & 59 & 6.4 & cT1c & $4+3=7$ & $\begin{array}{l}\text { LDR-BT } \\
(125 \mid)\end{array}$ & 53 & 22 & 145 Gy & 0.11 & 6 years & VMAT \\
\hline $\begin{array}{l}\text { Cooper } \\
\text { et al. }\end{array}$ & 2018 & 59 & 10.3 & cT2 & $3+4=7$ & $\begin{array}{c}\text { LDR-BT } \\
(125 \mid)\end{array}$ & 85 & 31 & 145 Gy & 2 & 11 years & ADT \\
\hline Our case & 2020 & 64 & 7.38 & cT2a & $\begin{array}{c}4+3=7 \\
\text { Ductal } \\
\text { adenoma }\end{array}$ & $\begin{array}{c}\text { LDR-BT } \\
(125 \mid)\end{array}$ & 70 & 16 & 160 Gy & 0.4 & 2 years & $\begin{array}{c}\text { Excised + } \\
\text { HDR + EBRT } \\
+ \text { ADT }\end{array}$ \\
\hline
\end{tabular}

N.A. - not available, BT - brachytherapy, EBRT-external beam radiation therapy, LDR - low-dose-rate, VMAT-volumetric modulated arc therapy, ADT-androgen deprivation therapy, HDR - high-dose-rate 
ed in the disappearance of the perineal mass. However, when the perineal mass progresses to castration-resistant prostate cancer and there is a local recurrence, the patient can suffer from localized pain and urinary symptoms. The use of local radiation therapy can prevent any potential future complication and symptoms. Moreover, recent studies $[18,19]$ have reported that radiation therapy for the primary site of metastatic prostate cancer can improve overall survival. Some other studies have described that radiation therapy for a metastatic site can improve the patients' prognosis $[20,21]$. Still, these results remain controversial. However, considering these outcomes, we expect that our radiation therapy for the perineal mass may have had a favorable influence on the patient's clinical outcome. There is no standard therapy for this kind of rare complication, therefore, each patient must be examined individually for the best treatment approach.

The clinical course of patients with perineal recurrence is not clear. Moul et al. described 6 patients who presented with perineal recurrence after needle biopsy. The authors reported that distant metastases were discovered simultaneously or within 16 months in all patients, who died within a median of 36 months after the initial diagnosis [2]. The previously reported 2 cases with a perineal recurrence after LDR-BT showed a good response to VMAT and ADT [6,7]. However, the observation period was too short to determine their prognosis. Because of the extremely limited number of reported cases with a perineal recurrence after LDR-BT, it is difficult to determine the risk factors for poor prognosis.

\section{Conclusions}

The present case showed that biochemical failure after transperineal brachytherapy for prostate cancer should be considered as a perineal recurrence. DAC showed unusual sites of recurrence, such as the perineal and mediastinal nodes in the present case. Therefore, careful follow-up is needed after the treatment for DAC. The treatment with both EBRT and HDR-BT boost might be an effective option for treating a perineal recurrence of prostate cancer after brachytherapy.

\section{Disclosure}

The authors report no conflict of interest.

\section{References}

1. Mottet N, Bellmunt J, Bolla $\mathrm{M}$ et al. EAU-ESTRO-SIOG guidelines on prostate cancer. Part 1: Screening, diagnosis, and local treatment with curative intent. Eur Urol 2017; 71: 618-629.

2. Moul JW, Miles BJ, Skoog SJ et al. Risk factors for perineal seeding of prostate cancer after needle biopsy. J Urol 1989; 142: 86-88

3. Seipel AH, Delahunt B, Samaratunga H et al. Ductal adenocarcinoma of the prostate: histogenesis, biology and clinicopathological features. Pathology 2016; 48: 398-405.

4. Roach 3rd M, Hanks G, Thames Jr H et al. Defining biochemical failure following radiotherapy with or without hormonal therapy in men with clinically localized prostate cancer: recommendations of the RTOG-ASTRO Phoenix Consensus Conference. Int J Radiat Oncol Biol Phys 2006; 65: 965-974.

5. Volanis D, Neal DE, Warren AY et al. Incidence of needle-tract seeding following prostate biopsy for suspected cancer: a review of the literature. BJU Int 2015; 115: 698-704.

6. Cooper S, Pillinger T, Ahmed I et al. Perineal recurrence of prostate cancer post-brachytherapy. BJR Case Rep 2019; 5: 20180104.

7. Eppinga W, Vijverberg $\mathrm{P}$, Moerland $\mathrm{R}$ et al. Perineal recurrence of prostate cancer six years after trans-perineal brachytherapy. J Contemp Brachytherapy 2014; 6: 386-388.

8. Teh BS, Chou CC, Schwartz MR et al. Perineal prostatic cancer seeding following radioactive seed brachytherapy. J Urol 2001; 166: 212.

9. Labardini MM, Nesbit RM. Perineal extension of adenocarcinoma of the prostate gland after punch biopsy. J Urol 1967; 97: 891-893.

10. Emtage JB, Perez-Marrero R. Extension of carcinoma of prostate along perineal needle biopsy tract. Urology 1986; 27: 548-549.

11. Bostwick DG, Kindrachuk RW, Rouse RV. Prostatic adenocarcinoma with endometrioid features. Clinical, pathologic, and ultrastructural findings. Am J Surg Pathol 1985; 9: 595-609.

12. Tu SM, Lopez A, Leibovici D et al. Ductal adenocarcinoma of the prostate: clinical features and implications after local therapy. Cancer 2009; 115: 2872-2880.

13. Copeland JN, Amin MB, Humphrey PA et al. The morphologic spectrum of metastatic prostatic adenocarcinoma to the lung: special emphasis on histologic features overlapping with other pulmonary neoplasms. Am J Clin Pathol 2002; 117: 552-557.

14. Tu SM, Reyes A, Maa A et al. Prostate carcinoma with testicular or penile metastases. Clinical, pathologic, and immunohistochemical features. Cancer 2002; 94: 2610-2617.

15. Ellis CL, Epstein JI. Metastatic prostate adenocarcinoma to the penis: a series of 29 cases with predilection for ductal adenocarcinoma. Am J Surg Pathol 2015; 39: 67-74.

16. Lemberger RJ, Bishop MC, Bates $\mathrm{CP}$ et al. Carcinoma of the prostate of ductal origin. Br J Urol 1984; 56: 706-709.

17. Vale JA, Patel A, Ball AJ et al. Endometrioid carcinoma of the prostate: a misnomer? J R Soc Med 1992; 85: 394-396.

18. Boevé LMS, Hulshof MCCM, Vis AN et al. Effect on survival of androgen deprivation therapy alone compared to androgen deprivation therapy combined with concurrent radiation therapy to the prostate in patients with primary bone metastatic prostate cancer in a prospective randomised clinical trial: data from the HORRAD Trial. Eur Urol 2019; 75: 410-418.

19. Parker CC, James ND, Brawley CD et al. Radiotherapy to the primary tumour for newly diagnosed, metastatic prostate cancer (STAMPEDE): a randomised controlled phase 3 trial. Lancet 2018; 392: 2353-2366.

20. Ost P, Bossi A, Decaestecker K et al. Metastasis-directed therapy of regional and distant recurrences after curative treatment of prostate cancer: a systematic review of the literature. Eur Urol 2015; 67: 852-863.

21. Connor MJ, Smith A, Miah S et al. Targeting oligometastasis with stereotactic ablative radiation therapy or surgery in metastatic hormone-sensitive prostate cancer: a systematic review of prospective clinical trials. Eur Urol Oncol 2020; 3: 582-593. 